EPJ Web of Conferences 59, 06005 (2013)

DOI: $10.1051 /$ epjconf/20135906005

(C) Owned by the authors, published by EDP Sciences, 2013

\title{
Development of laser ablation plasma by anisotropic self-radiation
}

\author{
Naofumi Ohnishi ${ }^{1, a}$ and Atsushi Sunahara ${ }^{2}$ \\ ${ }^{1}$ Department of Aerospace Engineering, Tohoku University, 6-6-01 Aramaki-Aza-Aoba, \\ Aoba-ku, Sendai 980-8579, Japan \\ 2 Institute for Laser Technology, 2-6 Yamadaoka, Suita, Osaka 565-0871, Japan
}

\begin{abstract}
We have proposed a method for reproducing an accurate solution of low-density ablation plasma by properly treating anisotropic radiation. Monte-Carlo method is employed for estimating Eddington tensor with limited number of photon samples in each fluid time step. Radiation field from ablation plasma is significantly affected by the anisotropic Eddington tensor. Electron temperature around the ablation surface changes with the radiation field and is responsible for the observed emission. An accurate prediction of the light emission from the laser ablation plasma requires a careful estimation of the anisotropic radiation field.
\end{abstract}

\section{INTRODUCTION}

High-temperature plasma ablated from laser-irradiated target is feasible for a source of extreme ultraviolet (EUV) light which is expected to be used in photolithography technology for the next generation of semiconductor devices. Many researchers have devoted their efforts to an accurate prediction of emission from such a target for improving conversion efficiency from the input laser energy to the EUV light by using numerical simulations [1]. Since the ablation plasma is in a highly nonequilibrium state, the emission depends on the atomic models employed in the simulation codes. In addition, radiation transfer process should be treated properly in the optically-thin ablation flow for an assessment of angular distribution of the EUV light.

Anisotropic radiation generally brings a huge computation of solving radiative transfer equation due to its multi-dimensionality. Some attempts have been examined with a variable Eddington factor estimated nonlocally for achieving a low cost computation. We have also proposed an efficient method for unsteady radiating flow in which anisotropic radiation plays an important role [2]. Before the coupling computation, we estimated angular distribution of emitted EUV light by ray-tracing method as a postprocess using a flowfield solution of laser-ablated tin target computed by the conventional radiation transfer method. Although the radiative flux measured at a distance is almost isotropic when the EUV emission is most intense during the laser pulse, the flux perpendicular to the target surface is enhanced due to the nonuniformity of the ablation plasma after the peak emission.

In this study, we focus on the accurate prediction of the emission from laser ablation plasma using our radiation hydrodynamics code and discuss about the importance of anisotropic treatment of radiation transfer through comparison between the conventional method and the proposed one. Moreover, we present the effect of the anisotropic radiation field on the dynamics of the ablation plasma and the resultant EUV emission through radiation hydrodynamics simulations coupling with new closure model by use of Monte-Carlo method.

\footnotetext{
ae-mail: ohnishi@rhd.mech.tohoku.ac.jp
}

This is an Open Access article distributed under the terms of the Creative Commons Attribution License 2.0, which permits unrestricted use, distribution, and reproduction in any medium, provided the original work is properly cited. 


\section{EPJ Web of Conferences}

\section{NUMERICAL MODELING}

Axisymmetric simulations of laser-produced plasma are conducted by one-fluid and two-temperature radiation hydrodynamics code. Governing equations for this code consist of Euler equations with energy sources of laser absorption, classical electron thermal conduction, and radiative interaction coupled with radiation transfer equation. Material property is characterized by non-LTE opacities and realistic EOS in the code [3].

In particular, numerical method for solving the radiation transfer equation is described below. We solve 0th and 1st moment equations instead of the original transfer equation for multi-grouped energy in x-ray range that significantly affects dynamics of laser ablation plasma;

$$
\begin{gathered}
\frac{\partial E^{v}}{\partial t}+\nabla \cdot \boldsymbol{F}^{\nu}=4 \pi \eta^{\nu}-c \chi^{v} E^{\nu}, \\
\frac{\partial \boldsymbol{F}^{v}}{\partial t}+c^{2} \nabla \cdot \mathbf{P}^{v}=-c \chi^{v} \boldsymbol{F}^{v},
\end{gathered}
$$

where $E, \boldsymbol{F}, \mathrm{P}, \eta$, and $\chi$ are the radiation energy density, the radiation flux, the radiation pressure tensor, the emissivity, and the opacity for the frequency $\nu$, respectively. When we assume that the Eddington approximation holds so that $\mathrm{P}^{v}=\mathrm{f}^{\nu} \mathrm{E}^{v}$, the Eddington tensor $\mathrm{f}^{v}$ is defined by

$$
\mathrm{f}^{v} \equiv \frac{\int_{4 \pi} I^{v} \boldsymbol{\Omega} \boldsymbol{\Omega} d \boldsymbol{\Omega}}{\int_{4 \pi} I^{v} d \boldsymbol{\Omega}},
$$

where $I^{v}$ and $\boldsymbol{\Omega}$ are the specific radiation intensity and the solid angle, respectively. The moment equations can be discretized for time derivative as follows,

$$
\begin{gathered}
E_{i, j}^{n+1}=E_{i, j}^{n}+\Delta t\left[4 \pi \eta_{i, j}^{n}-c \chi_{i, j}^{n} E_{i, j}^{n+1}-\left(\nabla \cdot \boldsymbol{F}^{n+1}\right)_{i, j}\right], \\
\boldsymbol{F}_{l, m}^{n+1}=\frac{\boldsymbol{F}_{l, m}^{n}}{1+c \chi_{l, m}^{n} \Delta t}-\frac{c^{2} \Delta t}{1+c \chi_{l, m}^{n} \Delta t}\left[\nabla \cdot\left(\mathrm{f}^{n} E^{n+1}\right)\right]_{l, m},
\end{gathered}
$$

where $(l, m)=(i+1 / 2, j)$ for $z$-component, and $(l, m)=(i, j+1 / 2)$ for $r$-component with staggered grids in a cylindrical geometry. If the Eddington tensor approaches $(1 / 3) \mathrm{l}$, the above discretization corresponds to the $\mathrm{P}_{1}$ approximation. In the latter equation, components of the divergence term are described in the following cylindrical forms:

$$
\begin{gathered}
{\left[\nabla \cdot\left(\mathbf{f}^{v} E^{v}\right)\right]_{z}=\frac{1}{r} \frac{\partial}{\partial r}\left(r f_{r z} E\right)+\frac{\partial}{\partial z}\left(f_{z z} E\right),} \\
{\left[\nabla \cdot\left(\mathbf{f}^{v} E^{\nu}\right)\right]_{r}=\frac{1}{r} \frac{\partial}{\partial r}\left(r f_{r r} E\right)+\frac{\partial}{\partial z}\left(f_{r z} E\right)-\frac{f_{\theta \theta} E}{r},}
\end{gathered}
$$

where $f_{\alpha \beta}(\alpha, \beta=r, \theta, z)$ represents element of the Eddington tensor. In this study, the terms with the off-diagonal elements in these equations are approximated by

$$
\begin{aligned}
& \left.\frac{1}{r} \frac{\partial}{\partial r}\left(r f_{r z} E\right)\right|_{i+1 / 2, j} \sim \frac{1}{r_{i+1 / 2, j} \Delta r}\left[r_{i+1 / 2, j+1 / 2} f_{r z_{i+1 / 2, j+1 / 2}}^{n}\left(E_{i+1, j}^{n+1}+E_{i, j+1}^{n+1}\right)\right. \\
& \left.-r_{i+1 / 2, j-1 / 2} f_{r z i+1 / 2, j-1 / 2}^{n} \frac{1}{2}\left(E_{i+1, j-1}^{n+1}+E_{i, j}^{n+1}\right)\right]
\end{aligned}
$$




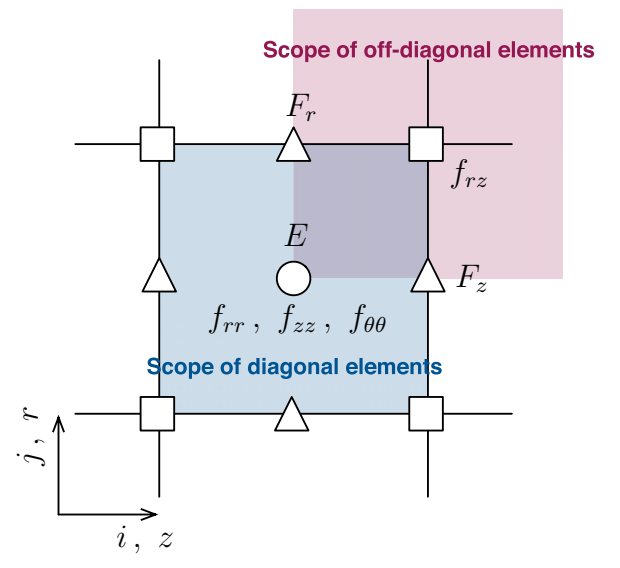

Figure 1. Definition point of each variable and scopes for Eddington tensor estimation.
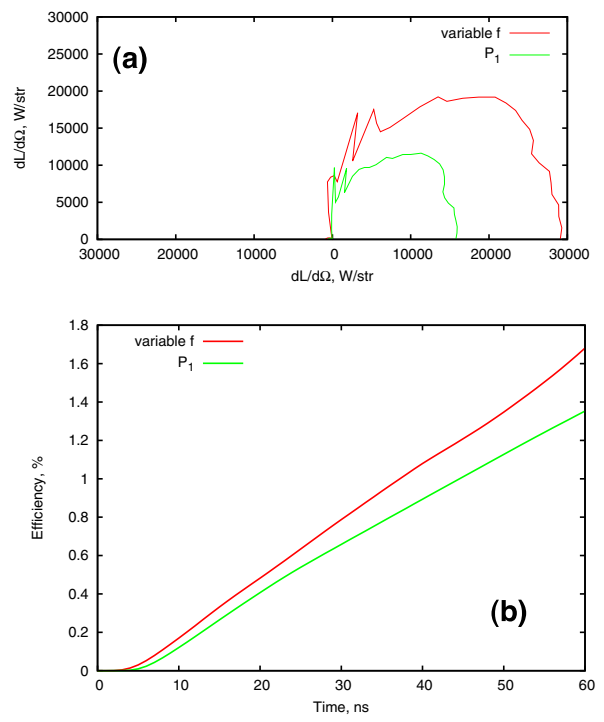

Figure 2. Comparison of (a) angular distribution of emitted EUV and (b) energy conversion efficiency to EUV.

$$
\left.\frac{\partial}{\partial z}\left(f_{r z} E\right)\right|_{i, j+1 / 2} \sim \frac{1}{\Delta z}\left[f_{r z i+1 / 2, j+1 / 2}^{n} \frac{1}{2}\left(E_{i, j}^{n+1}+E_{i+1, j+1}^{n+1}\right)-f_{r z i-1 / 2, j+1 / 2}^{n} \frac{1}{2}\left(E_{i-1, j}^{n+1}+E_{i, j+1}^{n+1}\right)\right] .
$$

Note that the discretization manner of these differential terms is not uniquely determined even with the 1st order accuracy, and we asymmetrically selected the cell-centered values for approximating the grid-vertex value to avoid the elimination of $E_{i, j}^{n+1}$ in the RHS.

Estimation of the Eddington tensor is a big issue for reproducing anisotropic radiation with moment equations. In the present paper, we estimate the elements of the Eddington tensor by tracing sample photons with Monte-Carlo manner. Initial position and propagation direction are randomly chosen with photon intensity $I_{p}(0)$ based on the emissivity of the given position, and $I_{p}(s)$ is diminished during the photon flight according to the opacity of the field, where $s$ is the photon path. The photon intensity is used by the estimation of the elements of the Eddington tensor as follows,

$$
\mathbf{f}=\frac{\int_{4 \pi} I \boldsymbol{\Omega} \boldsymbol{\Omega} d \boldsymbol{\Omega}}{\int_{4 \pi} I d \boldsymbol{\Omega}} \sim \frac{\sum_{p} I_{p} \boldsymbol{\Omega}_{p} \boldsymbol{\Omega}_{p}}{\sum_{p} I_{p}}
$$

The sampling for the summations is carried out when the photons enter the scope of each element of the Eddington tensor. For the diagonal elements, the scope is identical to the cell domain while for the offdiagonal elements, the rectangle scope is prescribed by centered points in the adjoining cells as shown in Fig. 1.

The Monte-Carlo method is useful but needs large number of samples for obtaining an accurate solution of radiation transfer equation. We may assume that the anisotropic feature, which appears in the Eddington tensor, does not drastically change as the fluid moves. Minor modification in each fluid time step may be allowable under the condition that the energy conservation is guaranteed by the moment equations. Small number of samples gradually changes the Eddington tensor in each time step. In this paper, initial Eddington tensor is estimated by 3 million sample particles. They are modified using 1000 


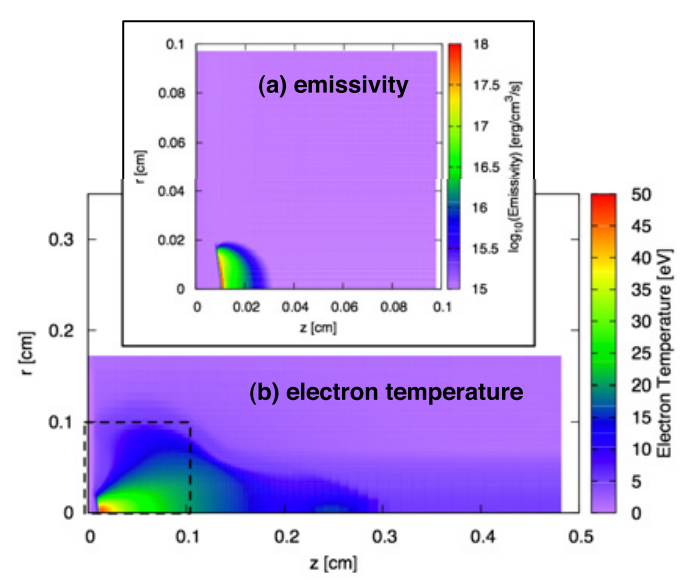

Figure 3. Contours of (a) EUV emission rate and (b) electron temperature with $\mathrm{P}_{1}$ approximation at $t=60 \mathrm{~ns}$.

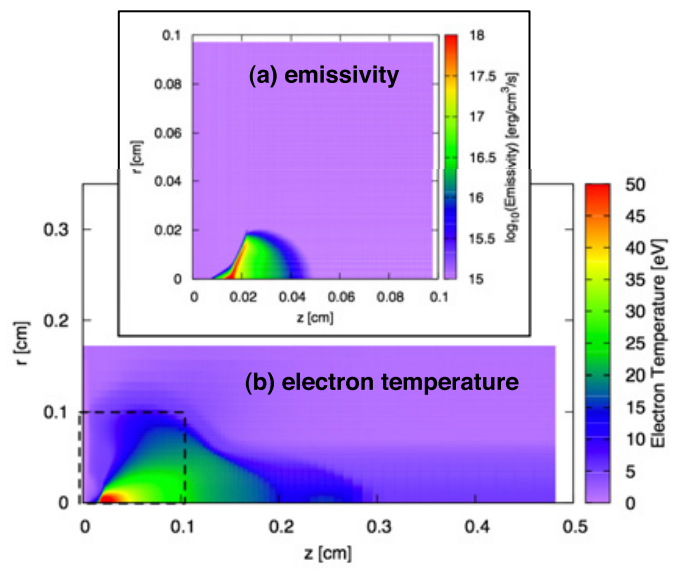

Figure 4. Contours of (a) EUV emission rate and (b) electron temperature with variable Eddington at $t=60 \mathrm{~ns}$.

sample particles for each fluid step via weighted averaging with the previous step value. We should emphasize that the Eddington tensor elements are not uniform in space even at the initial estimation.

\section{RESULTS AND DISCUSSIONS}

Numerical simulations of a planar tin target irradiated by $\mathrm{CO}_{2}$ laser of square pulse were conducted by our code with different radiation transfer methods. Laser intensity was set to $1.0 \times 10^{10} \mathrm{~W} / \mathrm{cm}^{2}$, and ray-tracing was one-dimensionally carried out for simplicity with Gaussian distribution of $100-\mu \mathrm{m}$ spot radius. The simulation results are compared between the case with constant Eddington tensor of $f=(1 / 3)$ l and that with variable one estimated by Monte-Carlo method. Hereafter, we refer to $\mathrm{P}_{1}$ approximation for the former case and variable Eddington for the latter.

Figure 2 shows (a) angular distribution of instantaneous EUV light for a distant observer at $t=60 \mathrm{~ns}$ and (b) conversion efficiency from laser energy to EUV computed by ray-tracing postprocess. For the case of variable Eddington, the instantaneous luminosity is a factor 2 higher, and the obtained emission is more anistropic. The obtained energy conversion efficiency is over $10 \%$ higher than the $\mathrm{P}_{1}$ approximation case. This difference between the transfer models is so serious for an accurate quantitative prediction of the emitted EUV light, which is highly required for commercial applications.

Since the EUV emission occurs around laser ablation surface, the ablation structure directly affects the emission rate. Figures 3 and 2 are a comparison of the emission rate and the electron temperature contours. Dashed squares in the lower panels of the electron temperature correspond to areas of the upper panels of the emission rate. For the case of variable Eddington, the ablation plasma has higher temperature especially around the symmetry axis, and provides a strong emission at the ablation surface. In fact, localization of radiation energy leads to such high electron temperature around the symmetry axis. The transfer computation at the symmetry axis should be carefully carried out and is not highly reliable yet.

Treatment of optically thick regions is also an issue to be improved because the sample photons hardly escape from such regions, and then the resultant Eddington tensor easily includes statistical errors. The errors especially appear in cold materials at the start-up phase. 
Moreover, criterion of number of sample particles in the Monte-Carlo method should be verified by further investigations for obtaining more reliable Eddington tensor.

\section{CONCLUSION}

We have proposed a method for reproducing an accurate solution of low-density ablation plasma by properly treating anisotropic radiation. The Monte-Carlo method is employed for estimating relatively accurate variable Eddington tensor with small computational costs in each fluid time step. Radiation field from ablation plasma is significantly affected by the anisotropic Eddington tensor. Ablation plasma structure drastically changes with the anisotropic radiation field. This indicates that emission from laser ablation plasma should be predicted by including the effect of the anisotropic radiation field.

\section{References}

[1] K. Nishihara et al., Phys. Plasmas 15, 056708 (2008)

[2] N. Ohnishi et al., Journal of Physics: Conference Series 244, 022078 (2010)

[3] A. Sunahara et al., Journal of Physics: Conference Series 112, 042048 (2008) 